\title{
SECAFÉ Parte I: Modelamiento y simulación matemática en el secado mecánico de café pergamino
}

\author{
Alfonso Parra-Coronado ${ }^{1}$, Gonzalo Roa-Mejía ${ }^{2}$ \& Carlos E. Oliveros-Tascón ${ }^{2}$
}

\begin{abstract}
RESUMEN
Se implementó en lenguaje de programación Microsoft Visual Basic 6.0, dos programas de simulación matemática para el secado de café pergamino, tomando como base los modelos de Thompson y de la Universidad del Estado de Michigan (MSU), para lo cual se utilizaron los parámetros determinados en Cenicafé para la simulación matemática del secado de café pergamino. Los programas estiman el funcionamiento de cada uno de los secadores mecánicos de café existentes en Colombia. Se compararon los resultados obtenidos mediante simulación para cada uno de los secadores, con los resultados experimentales obtenidos en Cenicafé, concluyéndose que éstos predicen adecuadamente el secado del café pergamino. Con base en los buenos resultados de la evaluación de los programas, se implementó un único programa de simulación para el secado de café pergamino.
\end{abstract}

Palabras claves: secadores mecánicos, modelo de Thompson, modelo MSU

\section{SECAFÉ Part I: Modeling and mathematical simulation in the mechanical drying of parchment coffee}

\begin{abstract}
A Microsoft Visual Basic 6.0 parchment coffee drying program was implemented. Two separate programs, based on the Thompson and Michigan State University (MSU) models, using Cenicafé coffee parameters, were successfully tested for the evaluation of the performance of all mechanical coffee dryers existing in Colombia. The results obtained by means of simulation for each one of the dryers were compared with the experimental results obtained in Cenicafé, resulting in the conclusion that these suitably predict the drying of the coffee parchment. Based on the good results obtained, a unique simulation program was implemented for drying parchment coffee.
\end{abstract}

Key words: mechanical dryers, Thompson's model, MSU model

1 Universidad Nacional de Colombia, Facultad de Ingeniería, Dpto. de Ingeniería Civil y Agrícola, Bogotá D. C., Colombia. Fone: (571) 316-5430. Fax: (571) 316-5462. E-mail:aparrac@unal.edu.co

2 Ingeniería Agrícola, Centro Nacional de Investigaciones de Café, Cenicafé. Chinchiná, Caldas, Colombia. Fone: (576) 850-6550. E-mail: gonzalo.roa@cafedecolombia.com, carlos.oliveros@cafedecolombia.com 


\section{INTRODUCCIÓN}

Cuando el café pergamino después de cosechado se deja por más de $48 \mathrm{~h}$ con su contenido inicial de humedad (52 al $56 \%$ bh, por retrazo en el proceso de secado), o cuando este se almacena con contenidos de humedad superiores al $12 \%$ bh (debido a un proceso de secado deficiente), el riesgo de ser atacado por hongos y de ser contaminado con micotoxinas es muy alto. La contaminación con hongos (moho) y OTA, hace que el grano pierda su calidad (CFC, 1999), ocasionando su rechazo en los mercados nacionales e internacionales, lo cual afecta drásticamente los ingresos de los caficultores y del país.

En consideración a lo anterior, se plantea como principal tecnología (la más viable técnica y económicamente) el adecuado y oportuno proceso de secado de los granos, para lograr disminuir su contenido de humedad a niveles del $10-12 \%$ bh, que permitan su almacenamiento por periodos prolongados.

Aunque en Colombia se ha venido produciendo café tipo exportación utilizando sistemas de secado que aprovechan las propiedades sicrométricas del aire ambiente, es necesario tener en cuenta sus limitantes (Roa et al., 1999) e incentivar al caficultor para que utilice técnicas de secado más eficientes, como es el caso del secado mecánico o artificial convección forzada de aire -, con los cuales se puede obtener ventajas como la reducción de la mano de obra, disminución del tiempo de secado y conservación de la calidad, de tal manera que permita obtener con menos riesgo "café tipo exportación» que cumpla con los requisitos de calidad exigidos por el mercado internacional. En efecto, no solo para Colombia y su café sino para todo el mundo, para todos los granos, cada día aumentan proporcionalmente los sistemas de secado mecánico, por razones de disminución de riesgos, costos y mejor control de la producción.

Si se desea que los sistemas de secado de café, o de cualquier grano, sean eficientes, se debe conocer la manera de operarlos adecuadamente (espesor de la capa de grano, caudal y temperatura del aire apropiados). El conocimiento de estas condiciones de operación se pueden investigar por dos métodos diferentes, así:

1. Mediante estudios experimentales, lo cual es un procedimiento largo (de varios años), dispendioso y costoso (Alzate, 1992; Buitrago, 1991; Loewer et al., 1994; Montenegro, 1992; Rivera \& Vélez, 1997; Roa et al., 1999).

2. Mediante el desarrollo y aplicación de técnicas de simulación matemática del secado ejecutadas por computadores, por medio de los cuales se analizan las predicciones del comportamiento de los granos durante el proceso de secado y se efectúan las recomendaciones para el dimensionamiento y operación de los sistemas de secado. (Bakker-Arkema et al., 1974; Brooker et al., 1992; Cenkowski et al., 1993; Domínguez et al., 1983; Montoya, 1989 ; Parra, 1990; Parra, 1993; Roa et al., 1999 ; Thompson et al., 1968).

De acuerdo con lo anterior, se ha demostrado universalmente (Bakker-Arkema et al., 1974; Brooker et al., 1992; Parra, 1990; Rossi \& Roa, 1980; Thompson et al., 1968), que la simulación matemática del secado es una alternativa viable, tanto tecnológica como económicamente, razón por la cual se realizó esta investigación, con el propósito de generar conocimientos para la operación eficiente de los secadores mecánicos de café utilizados en Colombia.

\section{MATERIAL Y METODOS}

Esta investigación se llevó a cabo en los laboratorios de Ingeniería Agrícola del Centro Nacional de Investigaciones de Café, Cenicafé, municipio de Chinchina - Caldas. Se desarrollaron las siguientes etapas investigativas: 1) obtención de los datos experimentales necesarios para las validaciones de los modelos matemáticos, de los secadores que no contaban con esta información; 2) desarrollo de los programas de simulación, evaluación y aplicación de los modelos.

\section{Secadores utilizados comercialmente en Colombia para café pergamino}

La Figura 1 muestra los secadores mecánicos utilizados en Colombia para el secado de café pergamino, los cuales son de dos tipos (Roa et al., 1999): «secadores estáticos»y «secador de flujo continuo». Los secadores estáticos son: el secador con una sola cámara (secador de una sola capa) con inversión de la dirección del flujo de aire, el silo-secador "Cenicafé», el secador de dos pisos (secador de dos capas verticales) y el secador de tres pisos. El secador de flujo continuo es un «secador intermitente de flujos concurrentes, Cenicafé-IFC», evaluado comercialmente en la Central de Beneficio de Anserma. Con base en la manera como operan cada uno de estos secadores, se desarrollaron los programas de simulación matemática del secado para cada uno de ellos.

\section{Obtención de datos experimentales}

Teniendo en cuenta que el proceso de secado de granos se considera determinístico, se realizaron cuatro pruebas experimentales de secado de café pergamino para cada uno de los sistemas de secado, que no contaban con datos experimentales suficientes para la validación de los modelos de simulación, a saber:

- Secador de dos pisos (silo-secador vertical).

- Secador de tres pisos (dos formas de manejo: «tipo de manejo simultáneo» y «tipo de manejo secuencial»)

A continuación se especifican los registros de la información que se tomó en cada una de las pruebas:

\section{Información correspondiente al grano de café pergamino}

Contenido de humedad del grano: Se determinó el contenido de humedad del café al inicio de la prueba y a intervalos de tres o cuatro horas durante el proceso de secado, para lo cual se tomaron las muestras en los respectivos secadores (aproximadamente $25 \mathrm{~g}$ por muestra) y se llevaron a la estufa a $105 \pm 1{ }^{\circ} \mathrm{C}$ durante $16 \pm 0,5 \mathrm{~h}$ (norma ISO 6673 de 1982). Las muestras (cinco por nivel) se tomaron en el nivel inferior y superior de cada una de las capas de grano contenidas en cada uno de los pisos, cuatro de ellas 


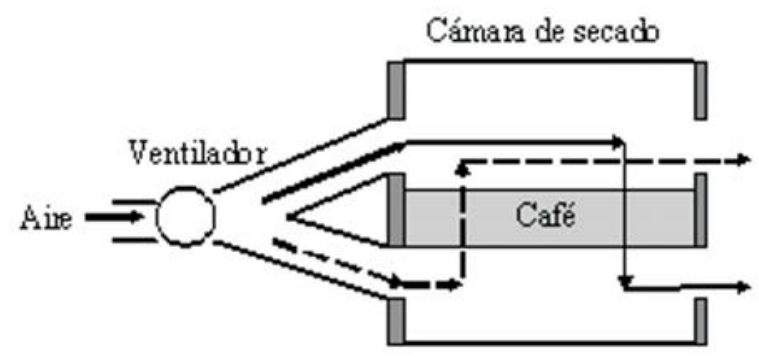

Secador de una sola capa, on inversión de la dirección de] flujo de aire

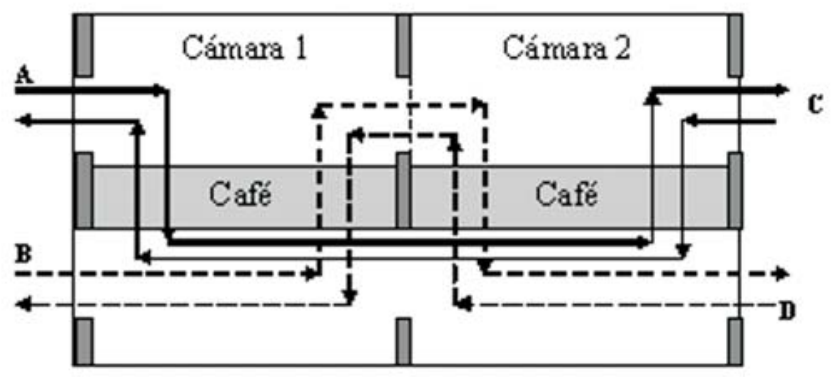

Secador de dos capas horizontales, "Silo-secador Cenicafe", ura de secado y otra de presecado con inversión de la dirección del flujo de aire

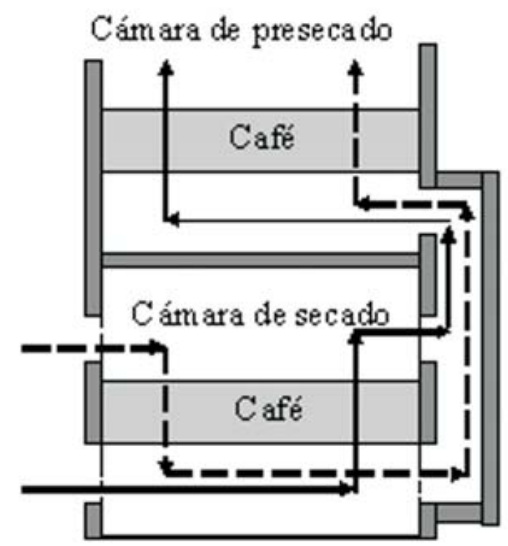

Secador de dos capas verticales con inversión de 1a dirección del flujo del aire solo en la capa inferior (cámar a de secado)

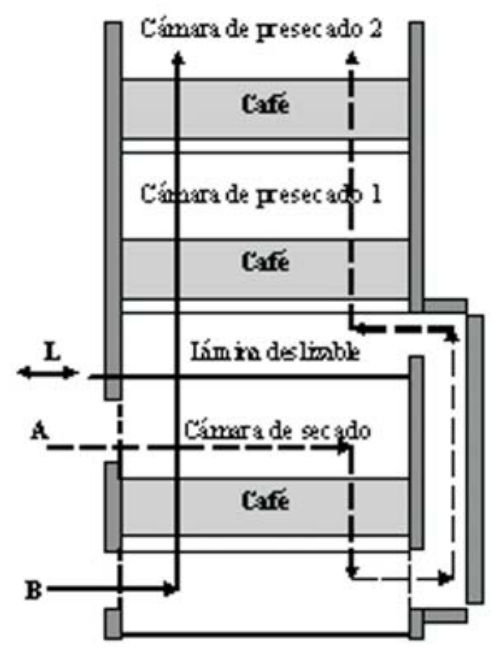

Secador de tres pisce verticales, on inversión de la dirección del flujo del aire en la capainferior

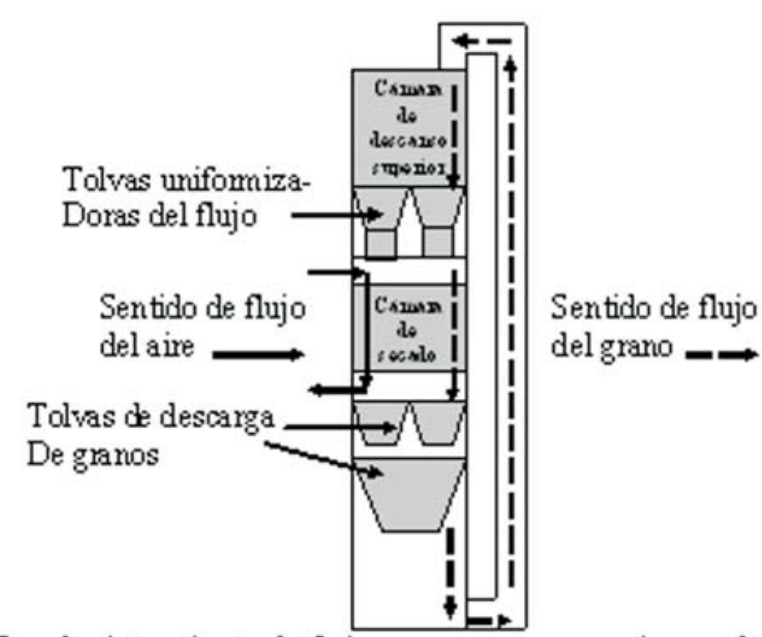

Secador intermitente de flujos concurrentes on cámaras de reposo supe rior e inferior

Figura 1. Secadores utilizados en Colombia para el secado mecánico de café pergamino

equidistantes $0,2 \mathrm{~m}$. en cada esquina y la quinta en el centro de la sección transversal.

Temperatura del grano: Se determinó la temperatura del grano al inicio de cada prueba y a intervalos de tres o cuatro horas durante el proceso de secado. Para la determinación de esta variable, cada una de las muestras tomadas en los respectivos secadores se llevó a un recipiente térmico - termo comercial -, el cual se acondicionó con un termómetro normal en la escala de -10 a $100{ }^{\circ} \mathrm{C}$; el mayor valor observado se consideró como la temperatura de la masa de grano.
Información correspondiente al aire de secado

Temperatura y humedad relativa del aire ambiente: Se utilizó un sistema electrónico para la adquisición de datos y termocuplas para medir la temperatura de bulbo seco (Series $3020 \mathrm{~T}$ Thermocouple digital data recorder). La temperatura de bulbo húmedo se determinó con un termómetro normal, cuyo bulbo se envolvió en una gasa humedecida. Tanto las termocuplas como el termómetro de bulbo húmedo, fueron colocados en el plenum del silo en la descarga del ventilador. Estas temperaturas se registraron cada hora. 
La humedad relativa se determinó a partir de las temperaturas de bulbo seco y bulbo húmedo.

Temperatura y humedad relativa del aire de secado: Se determinaron de manera similar a las temperaturas de bulbo seco y bulbo húmedo del aire ambiente, a la entrada al plenum del secador. La temperatura del aire de secado se fijó regulando el flujo de gas en el quemador.

\section{Información correspondiente al sistema de secado}

Para cada uno de los secadores utilizados en el presente experimento, se tomaron los datos correspondientes al área de la sección transversal del secador, altura de cada una de las capas de café y caudal de aire suministrado por el ventilador. Para la determinación del caudal de aire se tomaron los valores correspondientes a la velocidad media del aire utilizando un anemómetro digital - y al área de la sección a través de la cual fluye, correspondiente a la descarga del ventilador en el plenum. El caudal de aire en $\mathrm{m}^{3} \mathrm{~min}^{-1}$ se determinó mediante la Ecuación 1.

$$
\mathrm{Q}=\mathrm{V} \cdot \mathrm{A}
$$

donde:

$$
\begin{aligned}
& \mathrm{Q} \text { - caudal de aire de secado, } \mathrm{m}^{3} \mathrm{~s}^{-1} \\
& \mathrm{~V} \text { - velocidad del aire, } \mathrm{m} \mathrm{s}^{-1} \\
& \mathrm{~A} \text { - área perpendicular al flujo de aire, } \mathrm{m}^{2}
\end{aligned}
$$

\section{Desarrollo de los programas de simulación matemática del secado}

Los modelos de simulación matemática se implementaron en lenguaje de programación Microsoft Visual Basic 6.0, en un computador Pentium con capacidad de 128,0 MB RAM, tomando como base los modelos de Thompson y MSU (Michigan State University), para lo cual se utilizaron los parámetros determinados en Cenicafé para la simulación matemática del secado de café pergamino (Jaramillo, 1989; Montoya, 1989; Roa et al., 1999; Trejos, 1986).

Los modelos simulan el funcionamiento de cada uno de los secadores y estiman la capacidad dinámica y la eficiencia térmica del secador, así como la potencia requerida en el ventilador para vencer la resistencia de los granos al flujo del aire. Para el desarrollo de los modelos de los diferentes secadores, se tomaron como base las formas de operación de cada uno de ellos.

\section{Modelos de simulación matemática del secado}

Aunque se han desarrollado varios modelos para la simulación del secado de granos, los más utilizados son el modelo de Thompson (1968) y el modelo MSU desarrollado por Bakker-Arkema et al. (1974).

\section{Modelo de Thompson}

El modelo de Thompson (1968) es un modelo semiempirico, que fue desarrollado originalmente para simular el secado de maíz desgranado. En este modelo se considera la capa gruesa de granos, como constituida de capas delgadas de 2,5 cm. de espesor por capa, colocadas unas sobre otras. El enfoque básico para la simulación consiste en calcular el secado en una capa delgada de grano por medio de balances de calor y masa (modelo de equilibrio), y por medio de un proceso iterativo, aumentar varias capas delgadas para formar la capa gruesa de dimensiones iguales a las reales del secador. El secado de una capa delgada puede representarse por medio de ecuaciones (ecuación de secado en capa delgada), considerando los cambios de energía y humedad del grano y del aire.

El modelo predice el secado final de la capa de grano mediante el uso de la ecuación de secado en capa delgada, para lo cual se deben considerar las condiciones iniciales del aire y del grano. El balance de calor se completa al predecir las condiciones finales del aire y del grano. El modelo es flexible y permite integrar en él todas las variables y parámetros necesarios para expresar en ecuaciones el proceso de secado. El modelo completo es especialmente útil para el estudio de los diferentes sistemas de secado y para el diseño de equipos.

\section{Balance antes del secado}

El modelo de Thompson et al. (1968) realiza un balance de calor sensible antes de iniciar el proceso de secado, el cual consiste en la determinación de la temperatura de equilibrio entre el aire y el grano. La temperatura de equilibrio (Te) se determina a partir de la razón de humedad y temperatura del aire de secado ( $\mathrm{H}$ y $\mathrm{T}$ respectivamente) y de la temperatura y el calor específico del grano ( $\mathrm{Tg}$ y $\mathrm{Cp}$ respectivamente). La ecuación 2 es la resultante.

$$
\mathrm{Te}=\frac{(0.24+0.45 \mathrm{H}) \mathrm{T}+\mathrm{C}_{\mathrm{P}} \mathrm{Tg}}{0.24+0.45 \mathrm{H}+\mathrm{C}_{\mathrm{P}}}
$$

\section{Balance después del secado}

La simulación del secado de la capa de grano se realiza utilizando las ecuaciones características del grano, correspondientes a la «ecuación de contenido de humedad de equilibrio y de secado en capa delgada»; además se realizan los balances sicrométricos del aire de secado. El balance después del secado consiste en determinar la temperatura del aire y del grano (Tf) una vez haya transcurrido el intervalo de tiempo Dt; para ello se considera el «calor latente de vaporización del agua contenida en el grano», L. El grano habrá perdido humedad $(\mathrm{DH})$, la cual habrá sido removida por el aire (incrementando su razón de humedad a $\mathrm{H}_{\mathrm{f}}$ ). La Ecuación 3 determina el balance de calor después del secado.

$$
\mathrm{Tf}=\frac{\left(0.24+0.45 \mathrm{H}_{0}\right) \mathrm{Te}-\Delta \mathrm{H}(587.9+\mathrm{L}-\mathrm{Te})+\mathrm{C}_{\mathrm{P}} \mathrm{Te}}{0.24+0.45 \mathrm{H}_{\mathrm{f}}+\mathrm{C}_{\mathrm{P}}}
$$

En los secadores continuos de flujos concurrentes el grano y el aire se mueven en la misma dirección; se considera el grano en el secador como un conjunto de capas delgadas, con el aire de secado fluyendo hacia abajo por entre las capas de granos. Para cada intervalo de tiempo, una nueva capa de grano es localizada sobre la parte superior del secador y una capa es removida del fondo. El procedimiento para simplificar la simulación consiste en calcular los cambios de humedad de una capa a medida que ésta se desplaza a través del 
secador. Si el intervalo de tiempo es seleccionado adecuadamente, el aire de salida de una capa en la primera posición durante el primer periodo de tiempo, es el de entrada para la misma capa, la cual está en la segunda posición durante el segundo periodo de tiempo y así sucesivamente hasta obtener la profundidad deseada.

\section{Modelo MSU (Michigan State University)}

El modelo MSU, desarrollado por Bakker-Arkema et al. (1974), es un modelo teórico de no-equilibrio que se basa estrictamente en las leyes de transferencia de calor y de masa. El modelo MSU tiene el mismo enfoque básico que el modelo de Thompson; calcula las condiciones del aire y del grano en cada incremento de tiempo y espesor de capa realizando cuatro balances:

- Balance para la entalpía del aire

- Balance para la humedad del aire

- Balance para la entalpía del grano

- Balance para la humedad del grano

Estos balances originan ecuaciones diferenciales parciales que se resuelven simultáneamente por integración numérica, usando diferencias finitas, a partir de condiciones iniciales y de frontera (Bakker-Arkema et al., 1974). Con éste modelo se puede simular el funcionamiento de secadores estacionarios, de flujo cruzado, de flujos concurrentes y en contracorriente.

\section{Proceso de simulación en secadores estáticos utilizando el modelo MSU}

El modelo MSU realiza los balances de masa y energía sobre un volumen diferencial ( $\mathrm{Sdx}$ ), localizado en una posición arbitraria dentro del secador estacionario. Las variables desconocidas en el modelo son: el contenido de humedad y la temperatura de los granos individuales, la razón de humedad y la temperatura del aire de secado en el secador. El modelo para un secador estacionario consta de cuatro ecuaciones diferenciales parciales, provenientes de los balances de masa y energía del grano y del aire de secado y una ecuación de secado en capa delgada para el grano, a saber:

\section{Balance para la entalpía del aire}

Energía que sale = energía que entra - energía transferida por convección (Ecuación 4).

$$
\frac{\partial \mathrm{T}_{\mathrm{a}}}{\partial \mathrm{x}}=\frac{-\mathrm{ha}}{\mathrm{G}_{\mathrm{a}} \mathrm{C}_{\mathrm{a}}+\mathrm{G}_{\mathrm{a}} \mathrm{C}_{\mathrm{v}} \mathrm{H}}\left(\mathrm{T}_{\mathrm{a}}-\mathrm{T}\right)
$$

\section{Balance para la entalpía del producto}

Energía transferida = cambio en energía interna del producto - energía para evaporación (Ecuación 5).

$$
\frac{\partial T}{\partial t}=\frac{h a}{\rho_{P} C_{P}+\rho_{p} C_{w} M}(T a-T)+\frac{h_{f g}+C_{v}\left(T_{a}-T\right)}{\rho_{P} C_{P}+\rho_{p} C_{w} M} G a \frac{\partial H}{\partial x}
$$

\section{Balance para la razón de humedad del aire}

Humedad transferida $=$ humedad que entra - humedad que sale (Ecuación 6)

$$
\frac{\partial \mathrm{H}}{\partial \mathrm{x}}=\frac{\rho_{\mathrm{p}}}{\mathrm{G}_{\mathrm{a}}} \frac{\partial \mathrm{M}}{\partial \mathrm{t}}
$$

Ecuación de secado en capa delgada del producto, o de balance de humedad del grano

Deberá utilizarse una ecuación apropiada de capa delgada, la cual podrá tener la forma general de la Ecuación 7.

$$
\frac{\partial \mathrm{M}}{\partial \mathrm{t}}=\mathrm{f}\left(\mathrm{M}, \mathrm{Me}, \mathrm{M}_{0}, \mathrm{~T}, \ldots \mathrm{t}\right)
$$

Las ecuaciones 4, 5, 6 y 7 constituyen el modelo de simulación para un secador estacionario. Sin embargo, no se conoce una solución analítica del sistema de ecuaciones, por lo cual deberán usarse técnicas de solución aproximada, por diferencias o elementos finitos.

\section{Proceso de simulación en secadores de flujos concurrentes utilizando el modelo MSU}

De manera similar al modelo para secadores estacionarios, el modelo para secadores de flujos concurrentes realiza los balances de masa y energía sobre un volumen diferencial (Sdx). Las ecuaciones 8, 9 y 10 son resultantes de los respectivos balances.

$$
\begin{aligned}
& \frac{\mathrm{dT}_{\mathrm{a}}}{\mathrm{dx}}=\frac{-\mathrm{ha}}{\mathrm{G}_{\mathrm{a}} \mathrm{C}_{\mathrm{a}}+\mathrm{G}_{\mathrm{a}} \mathrm{C}_{\mathrm{v}} \mathrm{H}}\left(\mathrm{T}_{\mathrm{a}}-\mathrm{T}\right) \\
& \frac{\mathrm{dT}}{\mathrm{dt}} \frac{\mathrm{ha}}{\mathrm{G}_{\mathrm{p}} \mathrm{C}_{\mathrm{P}}+\mathrm{G}_{\mathrm{p}} \mathrm{C}_{\mathrm{w}} \mathrm{M}}\left(\mathrm{T}_{\mathrm{a}}-\mathrm{T}\right)-\frac{\mathrm{h}_{\mathrm{fg}}+\mathrm{C}_{\mathrm{v}}\left(\mathrm{T}_{\mathrm{a}}-\mathrm{T}\right)}{\mathrm{G}_{\mathrm{p}} \mathrm{C}_{\mathrm{p}}+\mathrm{G}_{\mathrm{p}} \mathrm{C}_{\mathrm{w}} \mathrm{M}} \mathrm{Ga} \frac{\mathrm{dH}}{\mathrm{dx}} \\
& \frac{\mathrm{dH}}{\mathrm{dx}}=\frac{\mathrm{G}_{\mathrm{p}}}{\mathrm{G}_{\mathrm{a}}} \frac{\mathrm{dM}}{\mathrm{dx}}
\end{aligned}
$$

Deberá utilizarse una ecuación apropiada de capa delgada (dM/dx), la cual podrá tener la forma general de la Ecuación 7.

La solución numérica de los modelos MSU requiere conocer las condiciones iniciales y de borde, o contorno, del grano y del aire de secado; dentro de las condiciones iniciales del grano y del aire se tienen: temperatura y contenido de humedad iniciales del grano; temperatura y razón de humedad del aire de secado a la entrada.

Las variables utilizadas en las ecuaciones 4 a 10 de los modelos MSU son las siguientes:

$\mathrm{a}$ - área específica del producto, $\mathrm{m}^{2} \mathrm{~m}^{-3}$

$\mathrm{C}_{\mathrm{a}}$ - calor específico del aire, $\mathrm{kJ} \mathrm{kg}^{-1}{ }^{\circ} \mathrm{C}^{-1}$

$\mathrm{C}_{\mathrm{p}}$ - calor específico del grano, $\mathrm{kJ} \mathrm{kg}^{-1}{ }^{\circ} \mathrm{C}^{-1}$

$\mathrm{C}_{\mathrm{v}}$ - calor específico del vapor, $\mathrm{kJ} \mathrm{kg}^{-1}{ }^{\circ} \mathrm{C}^{-1}$

$\mathrm{C}_{\mathrm{w}}$ - calor específico del agua, $\mathrm{kJ} \mathrm{kg}^{-1}{ }^{\circ} \mathrm{C}^{-1}$

$\mathrm{G}_{\mathrm{a}}$ - flujo másico de aire por unidad de área, $\mathrm{kg} \mathrm{h}^{-1} \mathrm{~m}^{-2}$

$\mathrm{G}_{\mathrm{p}}$ - flujo másico de grano por unidad de área, $\mathrm{kg} \mathrm{h}^{-1} \mathrm{~m}^{-2}$

$\mathrm{H}$ - razón de humedad del aire, $\mathrm{kg}$ de agua $/ \mathrm{kg}$ de aire seco

$\mathrm{h}$ - coeficiente de transferencia de calor por convección, $\mathrm{kJ} \mathrm{h}^{-1} \mathrm{~m}^{-2}{ }^{\circ} \mathrm{C}^{-1}$

$\mathrm{h}_{\mathrm{fg}}$ - calor de vaporización, $\mathrm{kJ} \mathrm{kg}^{-1}$

$\mathrm{M}$ - contenido de humedad local o promedio del grano, decimal bs 
$\mathrm{r}_{\mathrm{p}}$ - densidad en peso seco del grano, $\mathrm{kg} \mathrm{m}^{-3}$

$\mathrm{t}$ - tiempo, $\mathrm{h}$

$\mathrm{S}$ - sección del secador perpendicular al flujo del aire, $\mathrm{m}^{2}$

$\mathrm{T}_{\mathrm{a}}$ - temperatura del aire, ${ }^{\circ} \mathrm{C}$

$\mathrm{T}$ - temperatura del grano, ${ }^{\circ} \mathrm{C}$

$\mathrm{X}$ - coordenada dentro de la capa profunda de grano, $\mathrm{m}$

\section{Parámetros necesarios para los modelos de simulación del secado de café pergamino}

Para la simulación matemática del secado es necesario conocer las características físicas del grano y las propiedades del aire de secado. En cuanto a las características del grano se requiere conocer el peso específico aparente (densidad aparente); las ecuaciones de contenido de humedad en equilibrio, de calor específico, de calor latente de vaporización y de secado en capa delgada; del aire de secado se debe establecer la temperatura y humedad relativa (ó razón de humedad). Las ecuaciones y características del grano fueron determinadas en Cenicafé en diferentes trabajos de investigación (Jaramillo, 1989; López \& Ospina, 1990; Montoya, 1989; Rivera \& Vélez, 1997; Trejos, 1986).

\section{Coeficiente de transferencia de calor por convección entre el aire y el grano}

Brooker et al. (1992), presentan la ecuación 11 para el cálculo del coeficiente de transferencia de calor por convección en granos, el cual se utiliza en los modelos de simulación matemática de secado de la Universidad de Michigan (MSU).

$$
\mathrm{h}_{\mathrm{c}}=\mathrm{AC}_{\mathrm{a}} \mathrm{G}_{\mathrm{a}}\left(\frac{2 \mathrm{r}_{0} \mathrm{G}_{\mathrm{a}}}{\mathrm{C}+\mathrm{DT}}\right)^{\mathrm{B}}
$$

En donde:

$\mathrm{h}_{\mathrm{c}}$ - coeficiente de transferencia de calor por convección, $\mathrm{W} \mathrm{m}{ }^{-2}{ }^{\circ} \mathrm{K}^{-1}$

$\mathrm{r}_{0}$ - radio equivalente de la partícula (grano de café), $\mathrm{m}$

$\mathrm{C}_{\mathrm{a}}$ - calor específico del aire, $\mathrm{kJ} \mathrm{kg}^{-1}{ }^{\circ} \mathrm{K}^{-1}$

$\mathrm{G}_{\mathrm{a}}$ - flujo de aire, $\mathrm{kg} \mathrm{h}^{-1} \mathrm{~m}^{-2}$

$\mathrm{T}$ - temperatura del aire, ${ }^{\circ} \mathrm{K}$

$\mathrm{A}=0,2755 ; \mathrm{B}=-0,34 ; \mathrm{C}=0,06175 ; \mathrm{D}=0,000165$

\section{Calor específico del café pergamino}

Montoya (1989) utilizó el método de las mezclas, para determinar el calor específico del café pergamino en el rango de humedad del grano entre el 11 y el $45 \%$ bh, obteniendo la ecuación 12.

$$
\mathrm{C}_{\mathrm{P}}=1.3556+5.7859 \mathrm{CH}_{1}
$$

donde:

$$
\begin{aligned}
& \mathrm{Cp} \text { - calor específico, } \mathrm{kJ} \mathrm{kg}^{-1}{ }^{\circ} \mathrm{K}^{-1} \\
& \mathrm{CH}_{1} \text { - contenido de humedad, decimal, bs }
\end{aligned}
$$

\section{Contenido de humedad en equilibrio del café pergamino}

Trejos (1986) utilizó el método dinámico establecido por
Roa (Rossi \& Roa, 1980) para la determinación del contenido de humedad de equilibrio para café pergamino y encontró los coeficientes para la Ecuación 13.

$$
\mathrm{Me}=\left(\mathrm{P}_{1} \mathrm{HR}+\mathrm{P}_{2} \mathrm{HR}^{2}+\mathrm{P}_{3} \mathrm{HR}^{3}\right) \operatorname{Exp}\left[\left(\mathrm{Q}_{1} \mathrm{HR}+\mathrm{Q}_{2} \mathrm{HR}^{2}+\mathrm{Q}_{3} \mathrm{HR}^{3}\right) \mathrm{T}\right]
$$

donde:

Me - contenido de humedad de equilibrio del café pergamino, $\%$ bs.

HR - humedad relativa del aire, decimal

$\mathrm{T}$ - temperatura del aire, ${ }^{\circ} \mathrm{C}$

$\mathrm{P}_{1}=61.030848 \mathrm{Q}_{1}=-0.03049$

$\mathrm{P}_{2}=-108.37141 \mathrm{Q}_{2}=0.070114$

$\mathrm{P}_{3}=74.461059 \mathrm{Q}_{3}=-0.035177$

Esta ecuación es válida para un rango de temperaturas de 10 a $56{ }^{\circ} \mathrm{C}$ y humedades relativas entre 0 y $100 \%$.

\section{Calor latente de vaporización del agua en el café pergamino}

Trejos (1986), a partir de las isotermas de equilibrio higroscópico obtenidas para café pergamino y aplicando el método de Othmer, determinó la ecuación de calor latente de vaporización del agua en el café pergamino (Ecuación 14)

$$
\mathrm{L}=(2502.4-2.42958 \mathrm{~T})[1+1.44408 \operatorname{Exp}(-21.5011 \mathrm{M})]
$$

donde:

$$
\begin{aligned}
& \mathrm{L} \text { - calor latente de vaporización del café perga- } \\
& \text { mino, } \mathrm{kJ} \mathrm{kg}^{-1} \\
& \mathrm{~T} \text { - temperatura del café, }{ }^{\circ} \mathrm{C} \\
& \mathrm{M} \text { - contenido de humedad del café, decimal, bs }
\end{aligned}
$$

\section{Ecuación de secado en capa delgada}

López \& Ospina (1990) utilizaron el método dinámico para la determinación de los coeficientes (m, n y q) de la ecuación de secado en capa delgada de Roa (Ecuación 15), para contenidos de humedad del café desde $55 \%$ bh hasta la humedad de equilibrio. Los coeficientes de la ecuación fueron determinados para tres rangos de temperatura del aire de secado. La expresión es de la forma:

$$
\frac{\partial \mathrm{M}}{\partial \mathrm{t}}=-\mathrm{mq}(\mathrm{M}-\mathrm{Me})(\mathrm{Pvs}-\mathrm{Pv})^{\mathrm{n}} \mathrm{t}^{(\mathrm{q}-1)}
$$

donde:

$\mathrm{M}$ - contenido de humedad del grano en cualquier instante, $\%$, bs

Me - contenido de humedad de equilibrio, $\%$, bs

Pvs - presión de vapor de saturación, $\mathrm{kPa}$

$\mathrm{Pv}$ - presión de vapor parcial, $\mathrm{kPa}$

$\mathrm{T}$ - tiempo de secado, $\mathrm{h}$

En el presente trabajo y con base en simulaciones preliminares de secado, Roa determinó los siguientes parámetros $\mathrm{m}, \mathrm{n}, \mathrm{y}$ q para la ecuación unificada de secado en capa delgada, válida para los rangos $10-70{ }^{\circ} \mathrm{C}$ de temperatura y de 5 al $55 \%$ de humedad, bs: $m=0,0143 ; \mathrm{n}=0,87898$; $\mathrm{q}=1,06439$.

\section{Coeficiente de difusión de humedad}

Montoya (1989), obtuvo una ecuación para el coeficiente 
de difusión de humedad en el café pergamino, en función de la humedad y la temperatura del grano. La expresión es valida para contenidos de humedad menores o iguales a $50 \%$ bs. $(33,33 \%$ bh) (Ecuación 16)

$$
\mathrm{D}=4.1582 * 10^{-8} \operatorname{Exp}\left[(0.1346 \mathrm{Tg}+2.2055) \mathrm{M}-\frac{1184}{\mathrm{Tg}+273.16}\right]
$$

En donde:

$\mathrm{D}$ - Coeficiente de difusión de humedad, $\mathrm{m}^{2} \mathrm{~min}^{-1}$

$\mathrm{M}$ - Contenido de humedad media del grano, decimal, bs

$\mathrm{Tg}$ - Temperatura del grano, ${ }^{\circ} \mathrm{C}$

\section{Área específica del café pergamino}

Montoya (1989), considerando el grano de café como una esfera, obtuvo un área específica de $779,8 \mathrm{~m}^{2} \mathrm{~m}^{-3}$ para café pergamino, en el rango de contenido de humedad del 10\% al $25,6 \%$ bh, la cual fue aplicada en el modelo MSU para un secador de flujos concurrentes IFC.

\section{Densidad aparente del café pergamino}

Montoya (1989), encontró la Ecuación 17 para la densidad aparente del café pergamino, en función del contenido de humedad; esta es:

$$
\mathrm{DA}=365.884+2.7067 \mathrm{CH}_{2}
$$

En donde:

DA - densidad aparente, $\mathrm{kg} \mathrm{m}^{-3}$

$\mathrm{CH}_{2}$ - Contenido de humedad, \% bs.

\section{Evaluación de los programas de simulación matemática del secado}

Para determinar la conveniencia de los programas de simulación en la predicción del proceso de secado de café pergamino, se compararon los valores experimentales obtenidos en Cenicafé con los valores obtenidos mediante los modelos de simulación, utilizando el análisis gráfico de correspondencia de las tasas de secado (variación del contenido de humedad con respecto al tiempo) experimentales y simuladas; éste análisis permite observar gráficamente la precisión de cada uno de los modelos de simulación y las posibles etapas en las cuales el modelo subestima o sobreestima el proceso de secado. Estadísticamente se compararon las variaciones de humedad del grano, mediante correlación lineal entre lo experimental y lo simulado (prueba de dos colas).

Se validaron los programas desarrollados con base en los modelos de Thompson y MSU, comparando los resultados obtenidos mediante simulación con los valores experimentales encontrados en Cenicafé para los diferentes secadores, a saber: Secador de capa fija con y sin inversión del flujo del aire de secado (diez pruebas); Silo secador «Cenicafé» (cuatro pruebas); Secador de dos pisos o Silo-secador vertical (cuatro pruebas); Secador de tres pisos (cuatro pruebas); Secador intermitente de flujos concurrentes «Cenicafé-IFC» (trece pruebas).
Se compararon fundamentalmente los resultados correspondientes a las variaciones de los contenidos de humedad como función del tiempo de secado.

\section{RESULTADOS Y DISCUSIÓN}

\section{Programas de simulación matemática para secadores estáticos (modelo base: Thompson)}

Las simulaciones realizadas inicialmente para el carro secador modificado para secado mecánico y para el silo secador «Cenicafé» sin inversión del flujo de aire (utilizando el programa desarrollado con base en el modelo de Thompson), mostraron que los parámetros utilizados se ajustan muy bien al proceso real de secado, presentándose tan solo inconvenientes con la ecuación de secado en capa delgada. Esta ecuación originalmente se encontraba segmentada para tres diferentes intervalos de temperatura y sus parámetros $(\mathrm{m}, \mathrm{n}$ y q) estaban definidos para intervalos de confianza del $95 \%$.

Para que el modelo de simulación desarrollado funcionara correctamente, fue necesario realizar varias simulaciones con el fin de determinar los valores de los parámetros más adecuados (dentro de los intervalos de confianza) en cada uno de los segmentos de la ecuación de secado en capa delgada; una vez determinados estos parámetros, se procedió a determinar los valores unificados de los coeficientes $\mathrm{m}, \mathrm{n}$ y q para que la misma ecuación incluyera los tres intervalos de temperatura, dentro de todo el rango de humedad del café. El resultado de los trabajos de regresión dio los siguientes valores para la ecuación unificada de Roa (Ecuación 15):

$$
\mathrm{m}=0,0143 ; \mathrm{n}=0,87898 ; \mathrm{q}=1,06439
$$

\section{Programas de simulación matemática para secadores Cenicafé-IFC (modelo base: Thompson)}

Tomando como base el modelo de Thompson, se desarrolló el programa de simulación para un secador intermitente de flujos concurrentes «Cenicafé-IFC». Las simulaciones realizadas inicialmente utilizando los datos experimentales de Montoya (1989), mostraron que el modelo de simulación desarrollado se ajustaba muy bien al proceso real de secado, cuando se introducía el valor adecuado de incremento de tiempo (DELT) en la simulación; el inconveniente radicaba en encontrar el valor correcto de DELT. Para que el modelo de simulación desarrollado funcionara correctamente, sin necesidad de encontrar el DELT por tanteos sucesivos, se desarrolló una subrutina que lo estima automáticamente dentro del mismo programa de simulación (Subrutina CalDelt). Para la implementación de la subrutina se consideraron todos los parámetros que pudieran afectar el valor DELT, tales como:

- Características del aire de secado: caudal o flujo de aire y temperatura.

- Características del café pergamino: peso especifico aparente (densidad aparente), contenido de humedad y temperatura iniciales.

- Características del sistema: dimensiones de la sección de secado (sección transversal y altura), flujo de café 
pergamino y espesor de la capa delgada de grano.

Con base en un algoritmo reportado por Bakker-Arkema et al. (1974) que permite encontrar los valores del incremento de espesor de capa en función del flujo de aire, necesario para la estabilidad de la simulación en secadores de capa fija y de flujo cruzado en el modelo MSU, se determinó la ecuación 18 que estima el espesor de la capa delgada de grano para el secador de flujos concurrentes - IFC, la cual fue utilizada en la subrutina «CalDelt», obteniéndose excelentes resultados. La ecuación obtenida por Parra es:

$$
\frac{1}{\mathrm{EC}_{1}}=-3.03 \operatorname{Ln}\left(676.1035 \text { FluAir }_{1}-1798.26\right)+34.857
$$

donde:

$\mathrm{EC}_{1}$ - espesor de capa delgada de grano, $\mathrm{m}$

FluAir $_{1}$ - caudal o flujo de aire, $\mathrm{m}^{3} \mathrm{~min}^{-1} \mathrm{~m}^{-2}$

\section{Descripción general de los programas de simulación desarrollados}

Los programas implementados con base en el «modelo de Thompson», constan de un «programa o menú principal», mediante el cual se selecciona el tipo de secador que se desea simular (secadores estáticos de capa fija o secador intermitente de flujos concurrentes «Cenicafé-IFC») y varias subrutinas, cuyo número depende del tipo de secador. La «subrutina Thompson» es la base de todas ellas, y veintiuna subrutinas complementarias conforman el programa desarrollado para secadores estáticos de capa fija. El programa implementado con base en el modelo de Thompson para el secador intermitente de flujos concurrentes Cenicafé-IFC, está constituido por 11 subrutinas.

Los programas de simulación matemática para el secado de café pergamino con base en el «modelo de MSU», se desarrollaron con una estructura similar a los modelos basados en el modelo de Thompson. Constan de un «programa o menú principal», en el cual se selecciona el tipo de secador que se desea simular (secadores estáticos de capa fija o secador de flujos concurrentes IFC) y varias subrutinas, cuyo número depende del tipo de secador. La «subrutina MSU» (resuelve el sistema de ecuaciones para el modelo MSU) y la «subrutina Roa» (estima el contenido de humedad de equilibrio y simula el secado de la capa delgada de grano) son la base principal de todas ellas; veintidós subrutinas adicionales conforman el programa desarrollado con base en el modelo MSU para secadores estáticos de capa fija.

Se implementó otro programa para el «secador intermitente de flujos concurrentes Cenicafé-IFC», el cual consta de 22 subrutinas. Fue desarrollado con base en los modelos de Thompson y MSU, debido a que la ecuación de difusión, utilizada en el modelo MSU (Montoya, 1989) para estimar los estados de secado y de reposo del café pergamino, es válida solo para contenidos de humedad del grano menores o iguales a $50 \%$ bs $(33,33 \%$ bh. $)$. El programa desarrollado con base en el modelo de Thompson estima el estado de secado del café pergamino para cualquier contenido de humedad, pero no estima la variación de humedad dentro del grano del café, lo cual es un limitante para el dimensionamiento de estos secadores (no le permite calcular la longitud de las cámaras de reposo). El programa implementado utiliza el modelo de Thompson cuando el contenido de humedad promedio del café es superior a $50 \%$ bs; para valores inferiores de contenido de humedad, utiliza el modelo MSU.

\section{Validación de los programas de simulación matemática para secado de café pergamino}

Para determinar la bondad de los modelos de simulación en la predicción del proceso de secado de café pergamino, se compararon los valores experimentales obtenidos de los secadores reales operados en Cenicafé con los valores obtenidos mediante los programas de simulación. Para la validación se utilizó un análisis gráfico de correspondencia de las variaciones de los contenidos de humedad del café pergamino a lo largo del tiempo de secado, de las curvas de secado experimentales y simuladas; este análisis permitió observar gráficamente la precisión de los modelos de simulación, incluyendo las etapas en las cuales los modelos subestiman o sobreestiman el proceso de secado. Estadísticamente se compararon las curvas de secado del grano, mediante correlación lineal entre los resultados experimentales y los simulados.

Para los programas de simulación evaluados, el análisis gráfico de correspondencia de las curvas de secado experimentales y simuladas, así como el análisis estadístico para cada uno de los tipos de secadores, indican que los modelos desarrollados predicen muy bien el comportamiento del secado del café pergamino (en el $99 \%$ de los casos tc $<$ tt en la prueba de dos colas), para un rango de variación del contenido de humedad del café del 56,3 al 8,1\% bh. De igual manera, el coeficiente de determinación $\left\langle\mathrm{R}^{2}\right\rangle$ es superior a 0,93 en el $92 \%$ de los casos, (es mayor a 0,85 en el $99 \%$ de los casos), con un nivel de significación del 5\%, lo cual indica que por lo menos el $93 \%$ de la variabilidad de los datos obtenidos experimentalmente en el proceso de secado, es explicada por los modelos de simulación.

A continuación se presentan las gráficas comparativas entre los resultados experimentales y los simulados para una prueba típica de algunos de los secadores estudiados, así como su respectivo análisis.

La Figura 2 presenta los resultados obtenidos experimentalmente por Montenegro (1992) y mediante los programas de simulación desarrollados en este trabajo para un secador estático (sin cámara de presecado) con inversión del flujo de aire. En ella se observa que los programas predicen bien el proceso de secado en este tipo de secador, presentando un contenido de humedad mayor al experimental en $1,9 \%$ bs (es la máxima diferencia para la capa media) cuando se utiliza el programa desarrollado con base en el modelo de Thompson y de $2,8 \%$ bs (máxima diferencia para la capa superior) cuando se utiliza el programa desarrollado con base en el modelo MSU, para un tiempo de secado de $24 \mathrm{~h}$.

La predicción de los datos experimentales mediante el programa desarrollado con base en el modelo de Thompson está entre el 97 y 99\% para las tres capas consideradas durante el ensayo, mientras que para el programa desarrollado con base en el modelo MSU varía entre 95 y 97\%. Durante 

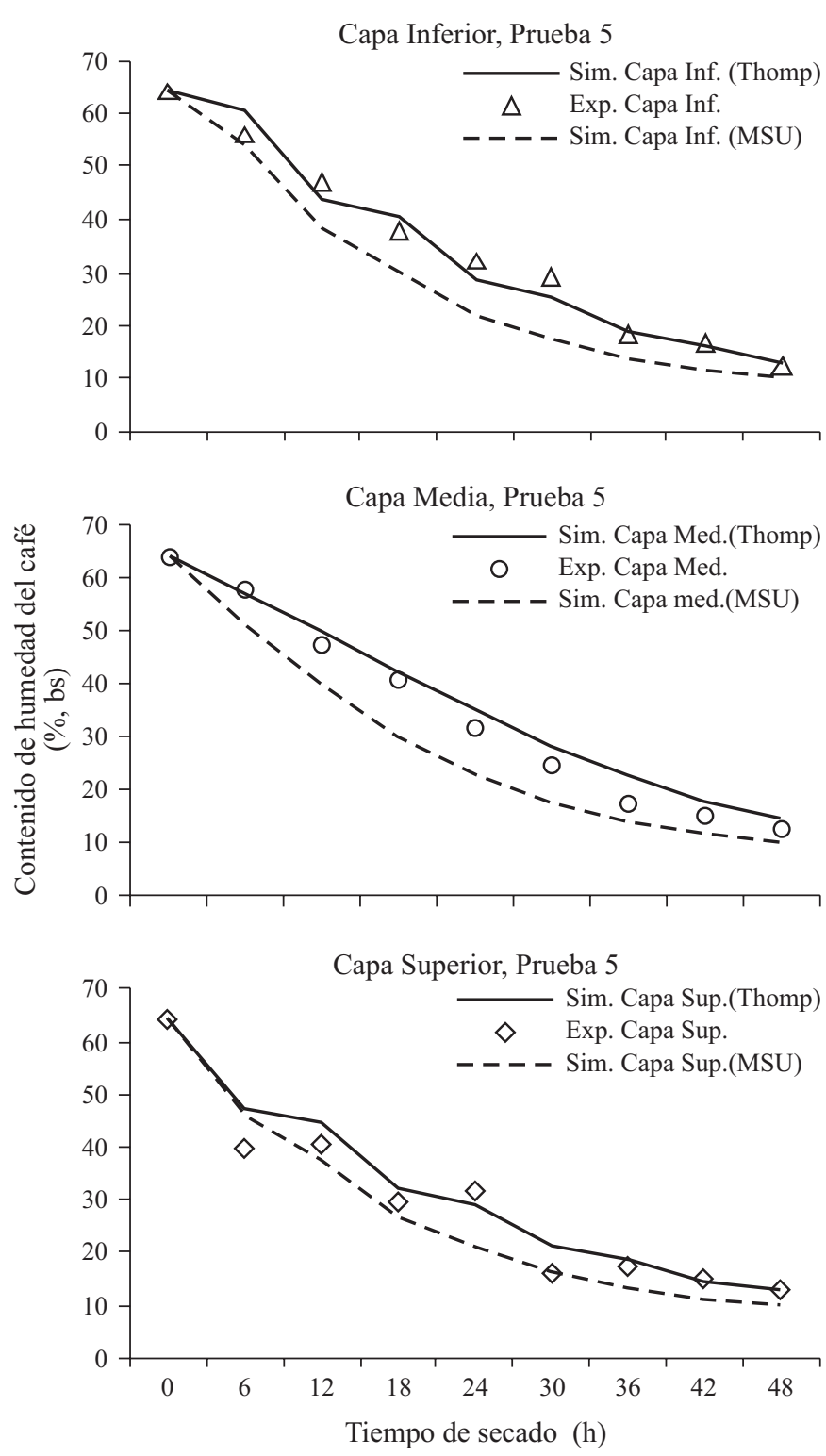

Figura 2. Curvas de secado para un secador estático sin cámara de presecado y con inversión del flujo de aire. Comparación de los resultados obtenidos experimentalmente (Montenegro, 1992) con los obtenidos por los programas de simulación desarrollados con base en los modelos de Thompson y MSU

el proceso, el café redujo su contenido de humedad promedio de 39,1 a $11,2 \%$ bh, cuando se utilizó una capa de café de $0,3 \mathrm{~m}$ y un caudal de aire de $7,6 \mathrm{~m}^{3} \mathrm{~min}^{-1} \mathrm{~m}^{-2}\left(456 \mathrm{~m}^{3} \mathrm{~h}^{-1}\right)$ a una temperatura de $39,5^{\circ} \mathrm{C}$.

Los resultados obtenidos para el Silo-secador «Cenicafé» muestran que los programas de simulación predicen bien el proceso de secado en este tipo de secador, presentando un contenido de humedad promedio - para el grano ubicado en la cámara de secado - mayor al experimental en 3,8 y 5,4\% bs, para los programas desarrollados con base en el modelo de Thompson y el modelo MSU respectivamente.

Para el grano ubicado en la cámara de presecado, el modelo de Thompson predice un contenido de humedad final promedio superior al experimental en $0,69 \%$ bs y el modelo MSU predice un contenido de humedad final promedio inferior al experimental en $2,47 \%$ bs. Los tiempos de secado son de 22 y 34 h para el grano en la cámara de secado y de presecado respectivamente. La predicción de los datos experimentales mediante el programa desarrollado con base en el modelo de Thompson está entre el 95 y 97\% para la cámara de secado y entre 92 y $97 \%$ para la cámara de presecado. Para el programa desarrollado con base en el modelo MSU varía entre 94 y $98 \%$ para la cámara de secado y entre 85 y $90 \%$ para la cámara de presecado.

Durante el proceso, el café redujo su contenido de humedad inicial promedio de 54,2 a 7,9 y $10,4 \%$ bh para el grano ubicado en las cámaras de secado y de presecado respectivamente, cuando se utilizó una capa de café de $0,4 \mathrm{~m}$ /cámara y un caudal de aire de $45,25 \mathrm{~m}^{3} \mathrm{~min}^{-1} \mathrm{~m}^{-2}$ $\left(149,34 \mathrm{~m}^{3} \mathrm{~min}^{-1}\right.$ ton $\left.^{-1}\right)$ a una temperatura de $50,6{ }^{\circ} \mathrm{C}$, con inversión del sentido del flujo de aire cada $6 \mathrm{~h}$.

En la Figura 3 se presentan los resultados obtenidos experimentalmente y mediante los programas de simulación desarrollados en este trabajo para un secador de tres pisos. En ella se observa que los programas de simulación predicen adecuadamente el proceso de secado en este tipo de secador. Para el grano ubicado en el primer piso (cámara de secado), los programas desarrollados con base en los modelos de Thompson y MSU presentan un contenido de humedad promedio mayor al experimental en 5,1 y $6,1 \%$ bs respectivamente.

Para el grano ubicado en el segundo piso (cámara de presecado 1), el modelo de Thompson predice un contenido de humedad final promedio superior al experimental en 5,4\% bs y el modelo MSU predice un contenido de humedad final promedio superior al experimental en $2,9 \%$ bs. Para el grano ubicado en el tercer piso (cámara de presecado 2), el modelo de Thompson predice un contenido de humedad final promedio superior al experimental en $4,9 \%$ bs y el modelo MSU predice un contenido de humedad final promedio inferior al experimental en 1,9\% bs. Los tiempos de secado son de 15, 22 y $29 \mathrm{~h}$ para el grano ubicado en el primero, segundo y tercer piso respectivamente.

La predicción de los datos experimentales mediante el programa desarrollado con base en el modelo de Thompson es en promedio de $99,98,8$ y 98,5\% para el primero, segundo y tercer piso respectivamente; para el programa desarrollado con base en el modelo MSU es de 99, 98 y 96\% para el primero, segundo y tercer piso respectivamente. Durante el proceso, el café redujo su contenido de humedad inicial promedio de 55,1 a $11,6 \%$ bh para el grano ubicado en los tres pisos (cámaras de secado y de presecado), cuando se utilizó una capa de café de $0,13 \mathrm{~m}$ por cámara y un caudal de aire de $31,41 \mathrm{~m}^{3} \mathrm{~min}^{-1} \mathrm{~m}^{-2}$ a una temperatura de $53,0{ }^{\circ} \mathrm{C}$, con movimiento del grano de una cámara a otra cada $4 \mathrm{~h}$ (Tipo de manejo secuencial).

La Figura 4 presenta los resultados obtenidos experimentalmente y mediante los programas de simulación implementados en éste trabajo, para un secador intermitente de flujos concurrentes «Cenicafé-IFC», con una altura de la columna de secado de $0,87 \mathrm{~m}$ y sección transversal de $0,7 \times 0,7 \mathrm{~m}$ (Prueba 2). En ella se observa que los dos programas de simulación predicen bien el proceso de secado en este tipo de secador. El programa desarrollado con base en el modelo de 


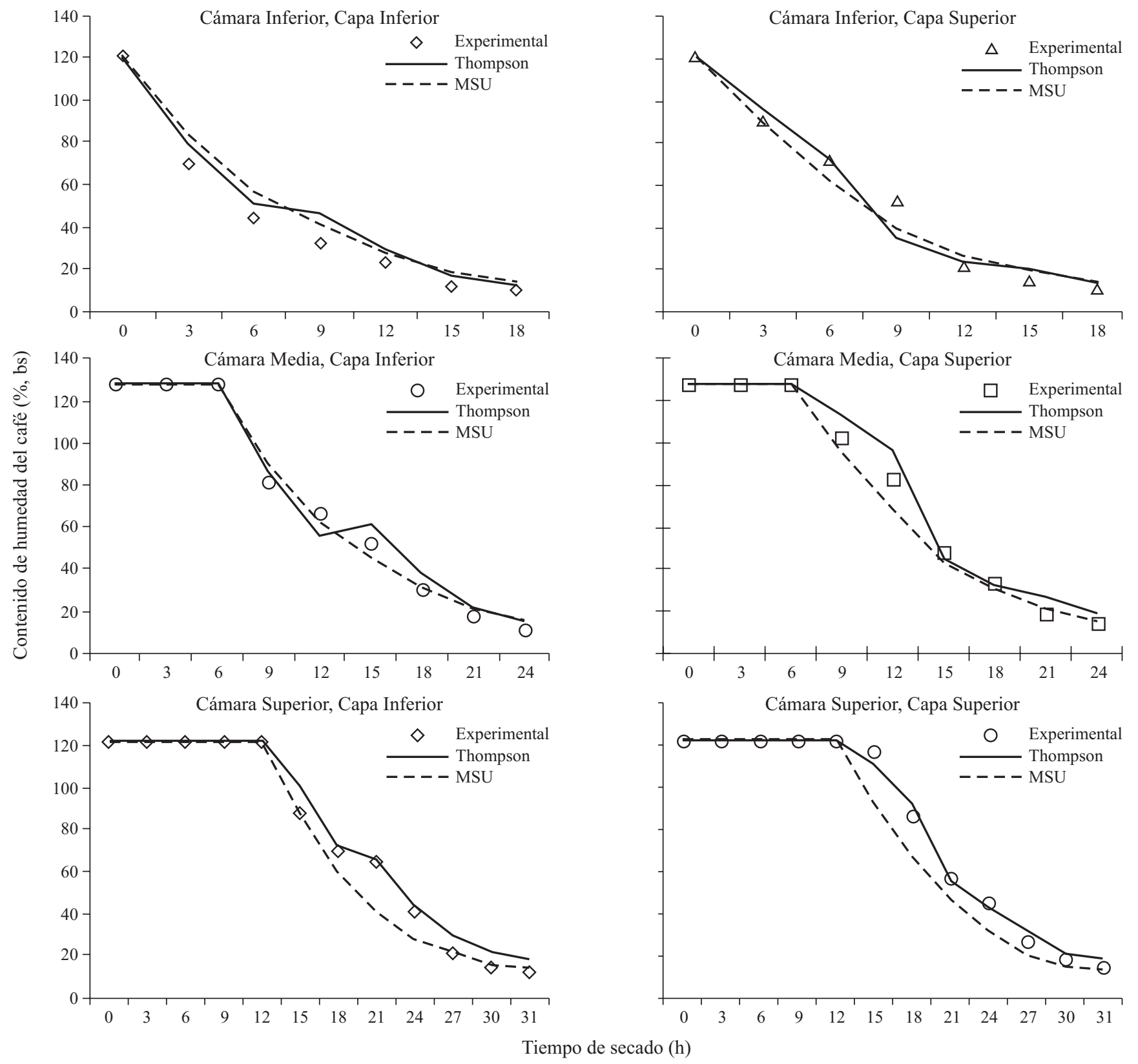

Figura 3. Curvas de secado para un Secador de tres pisos. Comparación de los resultados obtenidos experimentalmente (en este estudio) con los obtenidos por los programas de simulación desarrollados con base en los modelos de Thompson y MSU. Prueba 2. Manejo secuencial

Thompson predice un contenido de humedad final del grano inferior al experimental en $0,5 \% \mathrm{bs}$, mientras que el programa desarrollado con base en los modelos Thompson-MSU predice un contenido de humedad final del grano superior al experimental en $1,4 \%$ bs, para un tiempo de secado de 32,5 h. Durante el proceso, el café redujo su contenido de humedad promedio de 52,4 a $11,1 \%$ bh $(110$ a $12,5 \%$ bs $)$, cuando se utilizó un flujo de café de $1200 \mathrm{~kg} \mathrm{~h}^{-1} \mathrm{~m}^{-2}$ y un caudal de aire de $48 \mathrm{~m}^{3} \mathrm{~min}^{-1} \mathrm{~m}^{-2}\left(1.411,2 \mathrm{~m}^{3} \mathrm{~h}^{-1}\right)$ a una temperatura de $87^{\circ} \mathrm{C}$.

De acuerdo con los resultados obtenidos durante la validación de los programas de simulación de secado de café pergamino desarrollados con base en los modelos de Thompson y MSU, se puede concluir que éstos se ajustan muy bien a los resultados experimentales para secadores estáticos y

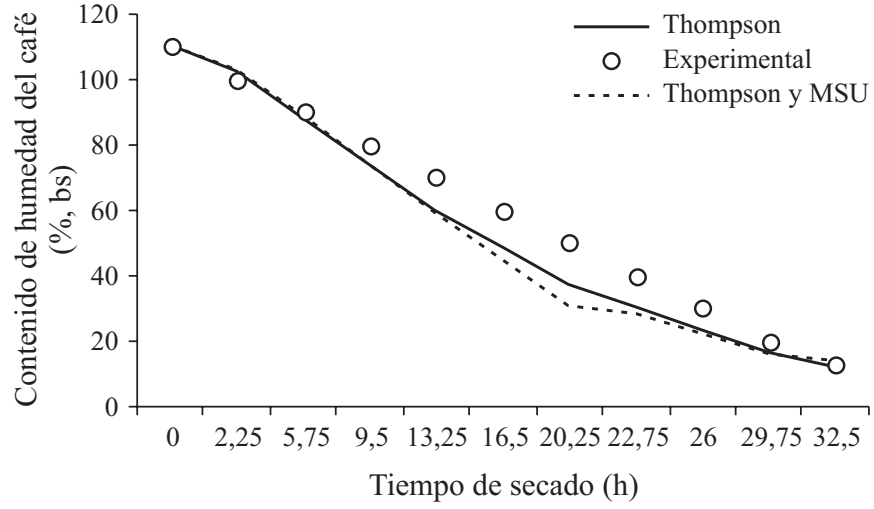

Figura 4. Curvas de secado para un secador intermitente de flujos concurrentes para café, "Cenicafé-IFC». Comparación de los resultados obtenidos experimentalmente (Montoya, 1989) con los obtenidos por los programas de simulación desarrollados. (Prueba 2) 
para el secador intermitente de flujos concurrentes «Cenicafé-IFC»; los modelos se pueden utilizar como una herramienta muy útil para predecir el comportamiento del grano durante el proceso de secado.

\section{Selección del programa de simulación más adecuado para} cada uno de los secadores utilizados para café pergamino

A pesar de que los dos programas implementados para los diferentes tipos de secadores estáticos (uno con base en el modelo de Thompson y otro con base en el modelo MSU) predicen muy bien el proceso de secado de café pergamino, estos mostraron diferencias en los tiempos totales de secado, las cuales pueden apreciarse en las Tablas 1, 2 y 3. Estas diferencias se deben fundamentalmente a los principios básicos de cada uno de los modelos utilizados (Thompson es un modelo de equilibrio, mientras que el modelo MSU es de no-equilibrio). También las diferencias se explican por los diferentes intervalos de simulación utilizados por cada uno de ellos con fines de estabilidad y convergencia de los programas (el modelo de Thompson utiliza incrementos de tiempo de simulación de una hora, mientras que el modelo MSU utiliza incrementos de $0,01 \mathrm{~h}$ ).

De la Tabla 1 se puede deducir que para los secadores estáticos sin cámara de presecado el programa que predice el secado del café pergamino con mayor precisión, teniendo en cuenta las diferencias absolutas entre los tiempos de secado simulados y experimentales, es el programa desarrollado con base en el modelo de Thompson, ya que presenta una diferencia absoluta del $8,6 \%$, frente a $16,9 \%$ del modelo MSU. La diferencia del 8,6\% del modelo de Thompson indica que los tiempos de secado encontrados mediante el programa de simulación, son $8,6 \%$ mayores que los reales, por lo cual los valores encontrados mediante simulación pueden ser corregidos por éste factor, si se desea hacer un uso exac-

Tabla 1. Tiempo de secado y diferencias absolutas entre los tiempos experimentales y simulados para Secadores Estáticos sin cámaras de presecado

\begin{tabular}{|c|c|c|c|c|c|c|c|}
\hline \multirow{3}{*}{ Prueba } & \multirow{2}{*}{\multicolumn{3}{|c|}{ Tiempo de secado (h) }} & \multicolumn{4}{|c|}{ Diferencia absoluta } \\
\hline & & & & \multicolumn{2}{|c|}{ Thompson } & \multicolumn{2}{|c|}{ MS U } \\
\hline & Exp & Thompson & MSU & h & $\%$ & h & $\%$ \\
\hline \multicolumn{8}{|c|}{ Carro secador modificado para secado mecânico } \\
\hline 1 & 24,0 & 26,0 & 27,2 & 2,0 & 8,3 & 3,2 & 13,3 \\
\hline 2 & 20,0 & 20,0 & 20,6 & 0,0 & 0,0 & 0,6 & 3,0 \\
\hline 3 & 24,0 & 26,0 & 29,0 & 2,0 & 8,3 & 5,0 & 20,8 \\
\hline 4 & 24,0 & 28,0 & 31,5 & 4,0 & 16,7 & 7,5 & 31,3 \\
\hline 5 & 24,0 & 26,0 & 26,5 & 2,0 & 8,3 & 2,5 & 10,4 \\
\hline 6 & 20,0 & 18,0 & 22,9 & 2,0 & 10,0 & 2,9 & 14,5 \\
\hline \multicolumn{8}{|c|}{ Secador estático con inversión del flujo de aire } \\
\hline 1 & 60,0 & 63,0 & 45,4 & 3,0 & 5,0 & 14,6 & 24,3 \\
\hline 4 & 60,0 & 67,0 & 50,7 & 7,0 & 11,7 & 9,3 & 15,5 \\
\hline 5 & 48,0 & 50,0 & 39,0 & 2,0 & 4,2 & 9,0 & 18,8 \\
\hline Cen $1^{*}$ & 24,0 & 26,0 & 28,0 & 2,0 & 8,3 & 4,0 & 16,7 \\
\hline Cen $2^{*}$ & 27,0 & 30,0 & 32,0 & 3,0 & 11,1 & 5,0 & 18,5 \\
\hline Cen $3^{*}$ & 27,0 & 30,0 & 31,1 & 3,0 & 11,1 & 4,1 & 15,2 \\
\hline \multicolumn{4}{|c|}{ Promedio Generale } & & 8,6 & & 16,9 \\
\hline
\end{tabular}

e Diferencias por exceso (tiempos encontrados mediante simulación mayores que los experimentales)

* Corresponde a datos de la cámara de secado del Silo-Secador «Cenicafé» únicamente to de ellos. No obstante, la mayor utilidad de las simulaciones matemáticas es, la mayor parte de las veces, para estudios de tendencias, visualizaciones, diseños, comparaciones, sensitividad, para lo cual los modelos desarrollados se consideran muy adecuados.

De la Tabla 2 se puede deducir que para el Silo-secador «Cenicafé» y para el Silo-secador vertical (dos pisos), el programa que predice el secado del café pergamino con mayor precisión, teniendo en cuenta las diferencias absolutas entre los tiempos de secado simulados y experimentales, es el programa desarrollado con base en el modelo MSU, ya que presenta una diferencia absoluta en el tiempo total de secado de $2,81 \%$, frente a $12,56 \%$ del modelo de Thompson. Los tiempos de secado encontrados mediante el programa de simulación desarrollado con base en el modelo MSU, exceden a los experimentales en 12,3 y $2,81 \%$ para las cámaras de secado y de presecado respectivamente, por lo cual los valores encontrados mediante simulación deben ser corregidos por estos factores, si se quiere hacer un uso exacto de ellos.

Tabla 2. Tiempo de secado y diferencias absolutas entre los tiempos experimentales y simulados para el Silo-secador "Cenicafé» y el Silosecador vertical

\begin{tabular}{|c|c|c|c|c|c|c|c|}
\hline \multirow{3}{*}{ Prueba } & \multirow{2}{*}{\multicolumn{3}{|c|}{ Tiempo de secado (h) }} & \multicolumn{4}{|c|}{ Diferencia absoluta } \\
\hline & & & & \multicolumn{2}{|c|}{ Thompson } & \multicolumn{2}{|c|}{ M S U } \\
\hline & $\operatorname{Exp}$ & Thompson & MSU & $\mathbf{h}$ & $\%$ & $\mathbf{h}$ & $\%$ \\
\hline \multicolumn{8}{|c|}{ Cámara de secado } \\
\hline 1 & 20,0 & 20,0 & 20,8 & 0,0 & 0,0 & 0,8 & 4,0 \\
\hline 2 & 18,0 & 19,0 & 20,8 & 1,0 & 5,6 & 2,8 & 15,6 \\
\hline 3 & 17,0 & 18,0 & 18,8 & 1,0 & 5,9 & 1,8 & 10,6 \\
\hline 4 & 21,0 & 23,0 & 25,0 & 2,0 & 9,5 & 4,0 & 19,1 \\
\hline \multicolumn{4}{|c|}{ Promedio ${ }^{\mathrm{e}}$} & & 5,2 & & 12,3 \\
\hline \multicolumn{8}{|c|}{ Cámara de presecado** } \\
\hline 1 & 24,0 & 27,0 & 25,2 & 3,0 & 12,5 & 1,2 & 5,0 \\
\hline 2 & 23,0 & 26,0 & 23,3 & 3,0 & 13,0 & 0,3 & 1,3 \\
\hline 3 & 23,0 & 25,0 & 23,5 & 2,0 & 8,7 & 0,5 & 2,2 \\
\hline 4 & 25,0 & 29,0 & 25,7 & 4,0 & 16,0 & 0,7 & 2,8 \\
\hline \multicolumn{4}{|c|}{ Promedio ${ }^{\mathrm{e}}$} & & 12,6 & & 2,8 \\
\hline
\end{tabular}

e Diferencias por exceso (tiempos encontrados mediante simulación mayores que los experimentales)

** Los tiempos de secado del grano ubicado en la cámara de presecado, corresponden al tiempo total de secado del café ubicado en el secador

De la Tabla 3 se puede deducir que para el Secador de tres pisos, el programa que predice el secado del café pergamino con mayor precisión, teniendo en cuenta las diferencias absolutas entre los tiempos de secado simulados y experimentales, es el programa desarrollado con base en el modelo MSU, ya que presenta una diferencia absoluta en el tiempo total de secado de 7,61\% frente a 10,88\% del modelo de Thompson. Los tiempos de secado encontrados mediante el programa de simulación desarrollado con base en el modelo MSU, exceden a los experimentales en 24,61 y $4,57 \%$ para el primero y segundo piso respectivamente, mientras que para el tercer piso son inferiores a los experimentales en 7,61\%. Si se desea utilizar de manera exacta los resultados de simulación, los tiempos de secado encontrados mediante el programa deben ser 
Tabla 3. Tiempo de secado y diferencias absolutas entre los tiempos experimentales y simulados para el secador de tres pisos

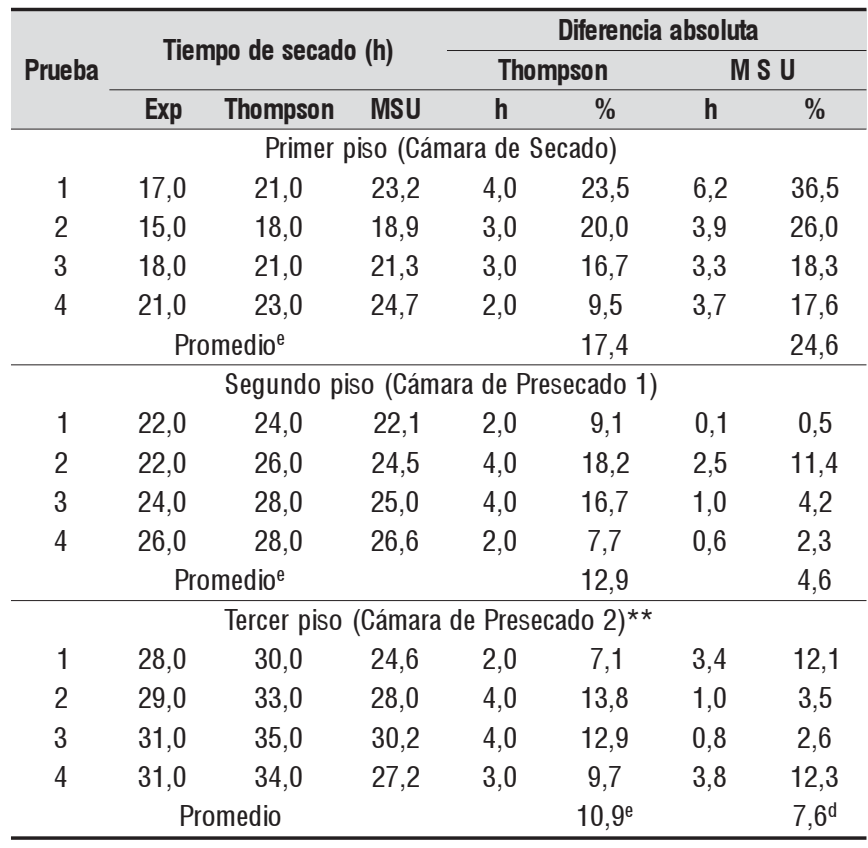

e Diferencias por exceso (tiempos encontrados mediante simulación mayores que los experimentales)

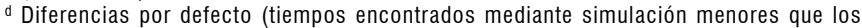
experimentales)

${ }^{* *}$ Los tiempos de secado del grano ubicado en el tercer piso (cámara de presecado 2), corresponden al tiempo total de secado del café ubicado en el secador

corregidos por los factores anteriores para cada una de las tandas (tanda 1: ubicada en el primer piso; tanda 2: ubicada en el segundo piso y tanda 3: ubicada en el tercer piso).

Para el Secador Intermitente de Flujos Concurrentes «Cenicafé-IFC», el mejor programa para predecir el secado del café pergamino es el implementado conjuntamente con los dos modelos de simulación (Thompson y MSU), ya que además de predecir el proceso de secado, simula además el proceso de reposo del grano (durante el reposo se estima el tiempo necesario para que ocurra la difusión de la humedad desde el interior del grano hacia la superficie del mismo), lo cual permite dimensionar los secadores "Cenicafé-IFC», e indicar la mejor manera de operarlos. El programa desarrollado con base en el modelo de Thompson predice muy bien el proceso de secado del grano, pero no considera el proceso de reposo, razón por la cual no puede estimar la longitud de la sección de reposo, presentando limitaciones para la simulación y el dimensionamiento de estos secadores.

\section{CONCLUSIONES}

1. Los resultados de simulación obtenidos con los programas implementados en la presente investigación, se ajustan muy bien a los resultados experimentales para todos los secadores estudiados.

2. Para los programas de simulación evaluados, el análisis gráfico de correspondencia de las tasas de secado experimentales y simuladas, así como el análisis estadístico para cada uno de los tipos de secadores, indican que estos mode- los predicen muy bien el comportamiento del secado del café pergamino, para un rango de variación del contenido de humedad del café del 56,3 al 8,1\% bh.

3. El coeficiente de determinación $« \mathrm{R}^{2} 》$ es superior a 0,93 en el $92 \%$ de los casos, con un nivel de significación del 5\%, lo cual indica que por lo menos el $93 \%$ de la variabilidad de los datos obtenidos experimentalmente en el proceso de secado, es explicada por los modelos de simulación.

4. Para los secadores estáticos sin cámaras de presecado, el modelo de Thompson es el que predice con mayor precisión la variación del contenido de humedad del grano a través del tiempo, así como el tiempo al final del secado, en tanto que para los secadores estáticos con cámaras de presecado, el modelo que predice con mayor precisión el proceso de secado es el MSU.

5. Para el Secador Intermitente de Flujos Concurrentes «Cenicafe-IFC», el mejor programa es el desarrollado con base en los dos modelos de simulación (Thompson y MSU), ya que con él se puede predecir el comportamiento del grano durante los estados de secado y de reposo, además de permitir el dimensionamiento (diseño) de este tipo de secadores

6. Con base en los resultados obtenidos, se procedió a implementar un único programa de simulación matemática para el secado de café pergamino en cada uno de los secadores existentes en Colombia.

\section{LITERATURA CITADA}

Alzate, J. G. Optimización operacional del silo-secador CENICAFE. Medellín: Departamento de Ingeniería Agrícola/ Universidad Nacional de Colombia, 1992. 240p. Tesis Ingeniero

Bakker-Arkema, F. W.; Lerew, L. E.; De Boer, S. F.; Roth, M. C. Grain drying simulation. Michigan: Research report from de Michigan State University East Lansing-MI. 1974. 80p.

Brooker, D. B.; Bakker-Arkema, F. W.; Hall, C. W. Drying and storage of grains and oilseeds. An AVI book, New York: Published by Van Nostrand Reinhold. 1992. 450p.

Buitrago, O. Implementación del secado mecánico de café en carros secadores. Bogotá: Departamento de Ingeniería Agrícola/Universidad Nacional de Colombia, 1991. 184p. Tesis Ingeniero

Cenkowski, S.; Jayas, D. S.; Pabis, S. Deep-Bed Grain Drying A Review of particular theories. Drying Technology, v.11, n.7, p.1553-1581, 1993.

CFC - Common Fund for Commodities. Enhancement of coffee quality through prevention of mould formation. Amsterdam: Appraisal Report, 1999, 44p.

Dominguez, J. Parra, A.; Villa, L. G. Simulación matemática y optimización del secado de productos agropecuarios con aire natural y energía solar. Revista Ingeniería e Investigación, v.2, n.3, p.57-62, 1983.

Jaramillo, B. G. Propiedades físicas del café pergamino. Bogotá: Departamento de Física/Universidad Nacional de Colombia, 1989. 190p. Tesis MSc

Loewer, O. J., Bridges, T. C.; Bucklin, R. A. On farm drying and storage systems. St. Joseph:American Society of Agricultural Engineers, ASAE Publication 9; Pam DeVore-Hansen Acquisitions; Books \& Journals, 1994. 560p. 
Lopez, J.; Ospina, J. Ecuación de capa delgada para el café pergamino. Cenicafé: Chinchiná, 1990. 183p. Informe de año sabático

Montenegro, J. Y. Secador eléctrico estático para café de baja capacidad, inversión de flujo y recirculación de aire. Bogotá: Departamento de Ingeniería Agrícola/Universidad Nacional de Colombia, 1992. 161p. Tesis Ingeniero

Montoya, E. C. Optimización operacional del secador intermitente de flujos concurrentes para café pergamino. Pereira: Facultad de Ingeniería Industrial/Universidad Tecnológica de Pereira, 1989, 116p. Tesis MSc

Parra, A. Potencial de secado de yuca con aire natural y energía solar en las regiones productoras de Colombia. Revista Latinoamericana ACOGRANOS, año 6, n.8, p.12-17, 1990.

Parra, A. Almacenamiento aireado de maíz en los Llanos Orientales. Revista Ingeniería e Investigación, v.8, n.29. p.5-13, 1993.
Rivera, O. L.; Velez P. A. Evaluación de una secadora de café de 12 arrobas (para pequeños cafeteros). Medellín: Corporación Universitaria Lasallista, Ingeniería de Alimentos, 166p, 1997. Trabajo Grado

Roa, G.; Oliveros, C. E.; Álvarez, J.; Ramírez, C.; Sanz, J. R.; Dávila, M. T.; Álvarez, J. R.; Zambrano, D. A.; Puerta, G. I.; Rodríguez, N. Beneficio ecológico del café. Chinchiná: CENICAFE, 1999, 300p.

Rossi, J. R.; Roa, G. Secagem e armazanamento de produtos agropecuários com uso de energia solar e ar natural. Sao Pablo: ACIESP, 1980, 293p.

Thompson, T. L.; Peart, R. M.; Foster, G. H. Mathematical simulation of corn drying - A new model. Transaction of the ASAE, v.11, n.4, p.582-586, 1968.

Trejos, R. R. determinación de las curvas de humedad relativa de equilibrio y del calor latente de vaporización del café pergamino y trillado. Cali: Universidad del Valle, Departamento de Procesos Químicos y Biológicos, 1986, 171p. Tesis Ingeniería 